\section{P259 SEEKING CLICKTHROUGHS: USING CELL PHONE APPLICATIONS IN PREVENTING STDS}

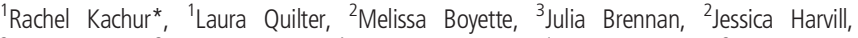
${ }^{2}$ Susan Jones, ${ }^{2}$ Joe Mclaughlin, ${ }^{4}$ Bozena Morawski, ${ }^{1}$ Alison Ridpath, ${ }^{2}$ Tracy Smith, ${ }^{2}$ Amanda Tiffany, ${ }^{5}$ Elizabeth Torrone, ${ }^{6}$ Kyle Bernstein. ${ }^{1}$ Centers for Disease Control and Prevention, Division of STD Prevention, Atlanta, USA; ${ }^{2}$ Alaska Department of Health and Social Services, Division of Public Health, Anchorage, USA; ${ }^{3}$ Tennessee Department of Health, Nashville, USA; ${ }^{4}$ Idaho Department of Health and Welfare, Boise, USA; ${ }^{5}$ US Centers for Disease Control and Prevention, Division of STD Prevention, Atlanta, USA; ${ }^{6}$ Centers for Disease Control and Prevention, Atlanta, USA

10.1136/sextrans-2019-sti.389

Background Rates of reported early syphilis in Alaska increased over 300\% between 2015 and 2018, with cases concentrated in men who have sex with men (MSM) that reside in the Anchorage/Mat-Su region. To better understand the epidemic, we surveyed at-risk Alaskan MSM about their syphilis knowledge, attitudes, and practices using a paper- and webbased survey. MSM were recruited through several avenues, including advertisements on two, popular geosocial-networking apps for gay, bisexual, and other MSM. We report on the relative efficiency in recruiting men through two different geosocial-networking apps.

Methods The geosocial-networking apps (App A and App B) were selected based on key-informant interviews. Identical ad text and imagery promoting the survey ran on each app for two weeks. The total number of times the ads were displayed (impressions), number of times users clicked on an ad (clicks), mean clickthrough rate (CTR; clicks/impressions), and costs for advertising on the two apps were estimated and compared.

Results App A had a total of 28,642 impressions, and 904 clicks (daily range: 5-191). The mean CTR for App A was 5.45 , at a cost of $\$ 0.32 /$ click. App B had a total of 681 impressions, and 57 clicks. The mean CTR for App B was 8.37 , at a cost of $\$ 12.28 /$ click. Of 119 survey responses, $59(50 \%)$ were online surveys, of which 32 (54\%) reported hearing about the survey through the geosocial-networking apps ad.

Conclusion Recruiting MSM for surveys and prevention interventions through mobile apps is productive and may be costefficient. However, not all apps produce the same results. In our survey, App B was over 38 times more costly per click than App A. Programs should routinely explore the effectiveness and associated costs with utilization of app-based advertising.

Disclosure No significant relationships.

\section{P260 RISK PERCEPTION, SAFER SEX PRACTICES, AND PREP ENTHUSIASM: EXPLORING PREP WITH BLACK AND MINORITY ETHNIC WOMEN IN THE UK}

${ }^{1}$ Sarah Nakasone*,${ }^{2}$ Ingrid Young, ${ }^{3}$ Claudia Estcourt, ${ }^{4}$ Josina Calliste, ${ }^{5}$ Paul Flowers, ${ }^{6}$ Jessica Ridgway, ${ }^{7}$ Maryam Shahmanesh. ${ }^{1}$ Chicago Center for HIV Elimination, Chicago, USA; ${ }^{2}$ University of Edinburgh, Usher Institute, Edinburgh, UK; ${ }^{3}$ Glasgow Caledonian University, School of Health and Life Sciences, Glasgow, UK; ${ }^{4}$ PrEPster, London, UK; ${ }^{5}$ University of Glasgow, MRC/CSO Social and Public Health Sciences Unit, Glasgow, UK; ${ }^{6}$ University of Chicago, Medicine, Chicago, USA; ${ }^{7}$ University College London, London, UK

10.1136/sextrans-2019-sti.390

Background Black and minority ethnic (BME) women in the UK remain disproportionally affected by HIV, comprising $75 \%$ of new diagnoses among UK women. Pre-exposure prophylaxis (PrEP) could offer an effective, autonomous, and discreet HIV prevention method for these women. However, PrEP uptake, where available, has been significantly limited. We explored possible reasons for this limited uptake.

Methods Using purposive sampling through community organizations, 32 in-depth semi-structured interviews were conducted with BME women living in London and Glasgow from JuneAugust 2018. Participants (ages 18-60) included women of varied HIV statuses to explore their knowledge of HIV and sexual health, perceptions of sexual risk, and attitudes to PrEP. Interviews were transcribed and an inductive thematic analysis was used to explore how PrEP knowledge and opinions intersected with wider understandings of safer sex

Results Women described extensive peer networks for sexual health advice that shaped their interactions with formal medical care. General HIV literacy was high, though PrEP-specific knowledge was low amongst non-HIV positive women. Participants expressed enthusiasm about PrEP for others but did not situate PrEP within their own safer sex narratives, often because of high levels of HIV stigma that caused women to ignore community risk factors and attribute infection to personal bad decisions. Alternately, some who were more familiar with PrEP believed it to be solely the domain of gay men. Many expressed concern that PrEP would undermine intimacy in their relationships by detracting from the shared responsibility of other HIV prevention practices, like joint testing.

Conclusion Low PrEP awareness and limited notions of candidacy contribute to limited PrEP uptake. For PrEP to be a useful tool for UK BME women, wider discussions of community risk are needed. HIV stigma and gendered responsibility for HIV prevention should be addressed. Existing peer networks should be harnessed to encourage nuanced messaging around these issues.

Disclosure No significant relationships.

\section{P262 PREP UTILIZATION AMONG YOUNG TRANSGENDER WOMEN, TRANSGENDER MEN, AND MSM IN AN URBAN COMMUNITY-BASED SETTING}

${ }^{1}$ Doreen Dankerlui* ${ }^{2}{ }^{2}$ Maureen Connolly, ${ }^{3}$ Christine Joseph, ${ }^{2}$ Tony Eljallad, ${ }^{4}$ Isadore DodardFriedman. 'Henry Ford Health System, Global Health, Detroit, USA; ${ }^{2}$ Henry Ford Health System, School-based and Community Health Programs, Detroit, USA; ${ }^{3}$ Henry Ford Health System, Public Health Sciences, Detroit, USA; ${ }^{4}$ University of Michigan, Ann Arbor, USA

\subsection{6/sextrans-2019-sti.391}

Background Little is known about factors influencing HIV pre-exposure prophylaxis (PrEP) utilization among young urban transgender women (TW), transgender men (TM) and men who have sex with men (MSM), who are disproportionately affected by HIV. This prospective pilot study explores PrEP adherence and persistence among these traditionally underserved communities in primary care clinics located in community centers serving LGBTQ youth.

Methods To examine factors related to PrEP outcomes, we established a cohort of PrEP-eligible young TW, TM and MSM at two clinics located in LGBTQ community centers and followed participants monthly. We measured tenofovir diphosphate levels at 12 weeks to assess adherence, defined as having a value $>700 \mathrm{fmol} /$ punch. Persistence was defined as picking up 2 refills during 12 weeks of follow up. Multivariate analysis will be used to describe associations between 